\title{
PARTITIONS OF VECTOR SPACES
}

\author{
AMER BEŠLAGIĆ
}

(Communicated by Andreas R. Blass)

\begin{abstract}
The following question is answered: If the real line is partitioned into countable sets, is there a Hamel basis that picks at most one element from each member of the partition?
\end{abstract}

In this note we answer the question from the abstract.

No special knowledge of set theory (or algebra) is required. All undefined notions and techniques used in the proof can be found in $[\mathrm{K}]$.

Let $\mathbf{V}$ be a vector space over a field $F$. In what follows we assume that $|\mathbf{V}|=\kappa$ is uncountable, and that $|F|<\kappa$.

If $X \subset \mathbf{V}$, then $\langle X\rangle$ is the subspace of $\mathbf{V}$ generated by $X$. Observe that $|\langle X\rangle| \leq \max (\omega,|X|,|F|)$ for any $X \subset \mathbf{V}$. Hence the dimension of $\mathbf{V}$ is $\kappa$.

The real line $\mathbf{R}$ is a vector space over the rationals. Any basis for this vector space is called a Hamel basis.

Since our result has the same proof for $\mathbf{R}$ as for any $\mathbf{V}$, we give proofs for uncountable vector spaces $\mathbf{V}$ (over fields whose cardinality is smaller than the cardinality of $\mathbf{V}$ ).

If $\mathscr{P}$ is a family of nonempty sets we say that a set $X$ is a transversal of $\mathscr{P}$ iff, for every $P \in \mathscr{P}, X$ intersects $P$ in exactly one point.

1. Theorem. Let $\mathscr{P}$ be a partition of $\mathbf{V}$ for which there is a $\lambda<\kappa$ such that, for each $P \in \mathscr{P},|P|<\lambda$. Then there is a transversal of $\mathscr{P}$ that generates $\mathbf{V}$.

Proof. Recall that $|\mathbf{V}|=\kappa>\omega$, so without loss of generality $\lambda \geq \max (\omega,|F|)$. Let $Y$ be a basis for $\mathbf{V}$. Let $\mathbf{A}$ be a partition of $\mathscr{P}$ such that, for each $\mathscr{A} \in \mathbf{A}$,

(1) $|\mathscr{A}|=|Y \cap(\cup \mathscr{A})|=\lambda^{+}$,

(2) $\langle Y \cap(\cup \mathscr{A})\rangle \backslash\{0\} \subset \cup \mathscr{A}$.

For $\mathscr{A} \in \mathbf{A}$, we show how to pick a transversal $X(\mathscr{A})$ of $\mathscr{A}$ with $X(\mathscr{A}) \subset \cup \mathscr{A}$ and $(Y \cap(\cup \mathscr{A})) \subset\langle X(\mathscr{A})\rangle$. Then $X=\cup\{X(\mathscr{A}): \mathscr{A} \in \mathbf{A}\}$ is as required.

Fix $\mathscr{A} \in \mathbf{A}$. List $Y \cap(\cup \mathscr{A})$ as $\left\{y_{\alpha}: \alpha<\lambda^{+}\right\}$and $\mathscr{A}$ as $\left\{A_{\alpha}: \alpha<\lambda^{+}\right\}$ (both listings one to one) so that $y_{0} \in A_{0}$ and, for each limit $\alpha, y_{\alpha} \in A_{\alpha}$.

Received by the editors February 3, 1989 and, in revised form, November 2, 1989.

1980 Mathematics Subject Classification (1985 Revision). Primary 04A20, 03 E50.

Key words and phrases. Partition, transversal, vector space. 
By induction on $\xi<\lambda^{+}$, we pick points $x_{\xi}$ so that

(a) $\forall \alpha<\lambda^{+}\left(\left|A_{\alpha} \cap\left\{x_{\xi}: \xi<\lambda^{+}\right\}\right| \leq 1\right)$ and

(b) $\left\{y_{\alpha}: \alpha<\lambda^{+}\right\} \subset\left\langle\left\{x_{\xi}: \xi<\lambda^{+}\right\}\right\rangle$,

thus showing that the required $X(\mathscr{A})$ exists.

Let $x_{0}=y_{0}$.

At stage $\xi$ : the points $\left\{x_{\eta}: \eta<\xi\right\}$ are already picked. If there is an $\alpha \leq \xi$ with

$$
A_{\xi} \cap\left(\left\langle\left\{y_{\alpha}\right\} \cup\left\{x_{\eta}: \eta<\xi\right\}\right\rangle \backslash\left\langle\left\{x_{\eta}: \eta<\xi\right\}\right\rangle\right) \neq \varnothing
$$

fix the minimal such $\alpha$ and pick any

$$
x_{\xi} \in A_{\xi} \cap\left(\left\langle\left\{y_{\alpha}\right\} \cup\left\{x_{\eta}: \eta<\xi\right\}\right\rangle \backslash\left\langle\left\{x_{\eta}: \eta<\xi\right\}\right\rangle\right) .
$$

Otherwise let $x_{\xi}=y_{0}$.

Observe that, for each $\xi,\left\{x_{\eta}: \eta<\xi\right\} \subset\left\langle\left\{y_{\eta}: \eta<\xi\right\}\right\rangle$, so if $\xi$ is a limit

$$
A_{\xi} \cap\left(\left\langle\left\{y_{\xi}\right\} \cup\left\{x_{\eta}: \eta<\xi\right\}\right\rangle \backslash\left\langle\left\{x_{\eta}: \eta<\xi\right\}\right\rangle\right) \neq \varnothing .
$$

In particular, for $\xi$ a limit, $x_{\xi} \notin\left\langle\left\{x_{\eta}: \eta<\xi\right\}\right\rangle$.

Now we show that $\left\{y_{\alpha}: \alpha<\lambda^{+}\right\} \subset\left\langle\left\{x_{\xi}: \xi<\lambda^{+}\right\}\right\rangle$. Assume not and let $\alpha$ be the least ordinal such that $y_{\alpha} \notin\left\langle\left\{x_{\xi}: \xi<\lambda^{+}\right\}\right\rangle$. Pick an ordinal $\mu>\max (\lambda, \alpha)$ so that $\left\{y_{\beta}: \beta<\alpha\right\} \subset\left\langle\left\{x_{\xi}: \xi<\mu\right\}\right\rangle$.

For $\xi \geq \mu$ let $S_{\xi}=\left\{y_{\alpha}+x_{\xi}+z: z \in\left\langle\left\{x_{\eta}: \eta<\xi\right\}\right\rangle\right\}$.

Since, for each $\zeta>\xi, S_{\xi} \subset\left\langle\left\{y_{\alpha}\right\} \cup\left\{x_{\eta}: \eta<\zeta\right\}\right\rangle \backslash\left\langle\left\{x_{\eta}: \eta<\zeta\right\}\right\rangle$, we have that $S_{\xi} \cap\left(\cup_{\zeta>\xi} A_{\zeta}\right)=\varnothing$, for otherwise $y_{\alpha} \in\left\langle\left\{x_{\eta}: \eta \leq \zeta\right\}\right\rangle$.

Since $0 \notin S_{\xi},(2)$ implies that $S_{\xi} \subset \cup_{\zeta<\lambda^{+}} A_{\zeta}$. Also, the points picked at limit stages are not in the span of the points picked before that stage, so $\left|S_{\xi}\right|=\lambda$, for $\xi>\mu$. But $\left|A_{\xi}\right|<\lambda$ so $S_{\xi} \cap\left(\cup_{\zeta<\xi} A_{\zeta}\right) \neq \varnothing$.

By the pressing down lemma, there is a $\gamma<\lambda^{+}$and a cofinal subset $T$ of limit ordinals less than $\lambda^{+}$, so that, for each $\xi \in T, S_{\xi} \cap A_{\gamma} \neq \varnothing$. So there are $\xi<\xi^{\prime} \in T$ with $S_{\xi} \cap S_{\xi^{\prime}} \neq \varnothing$.

Hence there are $z \in\left\langle\left\{x_{\eta}: \eta<\xi\right\}\right\rangle$ and $z^{\prime} \in\left\langle\left\{x_{\eta}: \eta<\xi^{\prime}\right\}\right\rangle$ with

$$
y_{\alpha}+x_{\xi}+z=y_{\alpha}+x_{\xi^{\prime}}+z^{\prime} \text {. }
$$

So $x_{\xi^{\prime}} \in\left\langle\left\{x_{\eta}: \eta<\xi^{\prime}\right\}\right\rangle$ which is a contradiction, since $\xi^{\prime}$ is a limit.

Note that the above theorem holds for $|F|=\kappa$ also, and that the theorem holds for $\kappa=\omega$ (this requires a different, but even easier, argument).

2. Theorem. There is a partition $\mathscr{P}$ of $\mathbf{V}$ such that, for each $P \in \mathscr{P},|P|<\kappa$, and no transversal of $\mathscr{P}$ generates $\mathbf{V}$.

Proof. Fix a basis $Y=\left\{y_{\alpha}: \alpha<\kappa\right\}$ for $\mathbf{V}$.

Define $P_{1}=\left\langle\left\{y_{0}, y_{1}\right\}\right\rangle$ and, for $\alpha>1, P_{\alpha}=\left\langle\left\{y_{\beta}: \beta \leq \alpha\right\}\right\rangle \backslash\left\langle\left\{y_{\beta}: \beta<\alpha\right\}\right\rangle$. Note that $\left|P_{\alpha}\right|<\kappa$ and $\left\langle\cup_{\beta<\alpha} P_{\beta}\right\rangle=\cup_{\beta<\alpha} P_{\beta}$, for $0<\alpha<\kappa$. 
We assume that $X$ is a transversal of $\mathscr{P}$ generating $\mathbf{V}$, and derive a contradiction.

Since $\left|X \cap P_{1}\right|<2$, without loss of generality $y_{0} \notin\left\langle X \cap P_{1}\right\rangle$. Let $y_{0}=$ $\sum_{i=1}^{n} a_{i} x_{i}$ where $a_{i} \neq 0, x_{i} \in X \cap P_{\alpha_{i}}$ for $1 \leq i \leq n$, and $\alpha_{1}<\alpha_{2}<\cdots<\alpha_{n}$. So $\alpha_{n}>1$, but then

$$
x_{n} \in\left\langle\left\{y_{0}\right\} \cup\left\{x_{i}: 1 \leq i<n\right\}\right\rangle \subset\left\langle\cup_{\beta<\alpha_{n}} P_{\beta}\right\rangle=\cup_{\beta<\alpha_{n}} P_{\beta},
$$

a contradiction.

3. Corollary. The Continuum Hypothesis is equivalent to the following statement:

There is a partition of the real line into countable sets such that each Hamel basis picks at least two elements from some member of the partition.

In closing let us notice that if the condition $\forall P \in \mathscr{P}(|P|<\lambda)$ in Theorem 1 is replaced by $\forall P \in \mathscr{P}(|P| \leq \lambda)$ then there is always a set $X$ that generates $\mathbf{V}$ such that $\forall P \in \mathscr{P}(|X \cap P| \leq 2)$. And if the above condition is furthermore relaxed to $\forall P \in \mathscr{P}(|P|<\kappa)$, then there is an $X$ with the above property iff $\kappa$ is regular.

\section{REFERENCE}

[K] K. Kunen, Set theory, North-Holland, Amsterdam, 1980.

Department of Mathematics, George Mason University, Fairfax, Virginia 22030 